\title{
因HAD
}

DOI: http://doi.org/10.22585/hospdomic.v4i4.116

\section{Repercusión en la capacidad funcional del ingreso en Hospitalización a Domicilio en pacientes mayores de 80 años}

\section{Impact of admission at Hospital at Home on the functional status in patients older than 80 years}

Esther Ferrer-Cobo', Ariadna Masferrer-Garcia², Noelia Quirant-Arellano², Lluïsa Moreno-Torrent ${ }^{2}$, Inmaculada García-Martínez ${ }^{2}$, Montserrat Ferre-Montoya ${ }^{2 \dagger}$, Mercedes Díaz-Gegúndez

1. Corporació de Salut del Maresme i la Selva, Hospital Comarcal de Blanes, Hospitalización a Domicilio, Blanes, España.

2. Corporació de Salut del Maresme i la Selva, Hospital Comarcal de Blanes,Hospitalización a Domicilio, Blanes, España.

3. Corporació de Salut del Maresme i la Selva, Hospital Comarcal Sant Jaume de Calella, Atención Domiciliaria Integral, Calella, España.

Correspondencia/Correspondence

Esther Ferrer-Cobo

eferrercobo@salutms.cat

Recibido/Received

14.09.2020

Aceptado/Accepted

14.09.2020
Conflicto de Intereses/Competing interest Las autoras declaran no tener ningún conflicto de intereses.

Agradecimientos/Acknowledgments

Al Dr Alberto Zamora por su lectura crítica.

CÓMO CITAR ESTE TRABAJO | HOW TO CITE THIS PAPER

Ferrer-Cobo E, Masferrer-Garcia A, Quirant-Arellano N, Moreno-Torrent L, García-Martínez I, Ferre-Montoya M, Díaz-Gegúndez M. Repercusión en la capacidad funcional del ingreso en Hospitalización a Domicilio en pacientes mayores de 80 años. Hosp Domic. 2020;4(4): 185-97. 


\section{RESUMEN}

Introducción: La hospitalización por enfermedad aguda en pacientes ancianos puede significar la aparición de deterioro funcional hospitalario (DFH). Por su elevada frecuencia y las graves consecuencias derivadas, analizamos el deterioro funcional en pacientes ingresados en una unidad de Hospitalización a Domicilio (HAD).

Método: Estudio descriptivo retrospectivo de pacientes $\geq 80$ años ingresados en HAD. Se obtuvieron variables demográficas y sociofamiliares, procedencia del ingreso, duración del ingreso previo e ingreso en HAD, variables clínicas y comorbilidad. Se recogió situación funcional basal, al ingreso, al alta y a los 3 meses según índice de Barthel (IB). Se excluyeron las estancias cortas, los paliativos, los fallecidos, aquellos con IB previo < 10 y los reingresos como motivo de alta.

Resultados: Se incluyeron 168 pacientes $\geq 80$ años, $52.4 \%$ hombres. El $71.4 \%$ procedentes de urgencias. Los pacientes institucionalizados presentaron peor resultado funcional. El 40,5\% presentó pérdida funcional (PF) al ingreso. Al alta, mejoraron $1.2 \%$, se mantuvieron el $59,3 \%$ y empeoraron el 39,4\%. La PF al alta es menor si la estancia hospitalaria es $\leq 2$ días y la estancia total $<7$ días. El uso de sonda vesical se asocia a peor resultado funcional al alta y se mantiene a los 3 meses.

Conclusiones: La HAD puede reducir el DFH si se acorta la estancia hospitalaria previa.

Palabras clave: pérdida funcional; Hospitalización a Domicilio; paciente geriátrico; deterioro funcional

\section{ABSTRACT}

Introduction: Hospitalization for acute illness in elderly patients may precipitate the appearance of hospital functional impairment (HFI). Due to its high frequency and the serious consequences derived, we analysed functional results in patients admitted to a Hospital at Home (HAH) unit.

Method: Retrospective descriptive study of patients $\geq 80$ years admitted to $\mathrm{HAH}$. We collected sociodemographic characteristics, source of referral, previous hospital stay and $\mathrm{HAH}$ stay, clinical assessment and comorbidity. Functional status previous, at admission, at discharge and after 3 months was collected according to the Barthel index (BI). Short stays, palliative care, deaths, those with a previous $\mathrm{BI}<10$, and readmissions as a reason for discharge were excluded.

Results: 168 patients $\geq 80$ years old, $52.4 \%$ men, were included. $71.4 \%$ admitted from the emergency department. Institutionalized patients presented worse functional results. $40.5 \%$ presented functional loss (FL) at admission. At discharge, they improved $1.2 \%$, remained $59.3 \%$ and worsened $39.4 \%$. The FL at discharge is lower if the previous hospital stay is $\leq 2$ days and the total stay $<7$ days. The use of bladder catheter is associated with a worse functional result at discharge and is maintained at 3 months.

Conclusions: HAH can reduce HFI if the previous hospital stay is shortened.

Keywords: functional decline; Hospital at Home; geriatric assessment; functional impairment 


\section{INTRODUCCIÓN}

La hospitalización por enfermedad aguda en personas mayores puede dar lugar a aparición de complicaciones no relacionadas con el motivo de ingreso o el tratamiento específico, así como a pérdida de la independencia (1-3).

El envejecimiento de la población obliga a los sistemas de salud a dar respuestas concretas a las necesidades de los pacientes ancianos. Además de los resultados de salud derivados del ingreso hospitalario (altas, defunciones, reingresos) es igualmente importante prestar atención al deterioro funcional asociado al ingreso, o Deterioro Funcional Hospitalario (DFH), definido como la pérdida de autonomía o capacidad en el funcionamiento físico que ocurre durante la estancia hospitalaria, por su elevada frecuencia y por las graves consecuencias que comporta, como son la peor evolución clínica, aumento de complicaciones, mayor estancia hospitalaria, mayor consumo de recursos sanitarios, aumento de los reingresos, aumento de la dependencia, la institucionalización, la sobrecarga del cuidador, así como el incremento de la mortalidad (1,3-10).

El deterioro funcional respecto a la situación basal (considerada habitualmente como la situación funcional 2 semanas antes del ingreso) se denomina deterioro funcional hospitalario global, que incluye el deterioro pre-hospitalización y el deterioro interhospitalario (2).

Diversos trabajos realizados para estudiar la evolución funcional de los pacientes mayores de 70 años ingresados por enfermedad aguda describen que entre el 31 y el $83.7 \%$ de los pacientes presentaron DFH global ${ }^{(3,6,64 \%}$ women, $24 \%$ nonwhite 10-13). En pacientes nonagenarios se ha descrito DFH en el $88 \%$ de los pacientes (14).

Algunos de estos estudios han seguido la evolución del deterioro funcional hasta 3 meses, padeciendo el 19\% de los mayores de 70 años deterioro en alguna actividad básica de la vida diaria (ABVD) (10). En los mayores de 90 años el deterioro funcional respecto a su situación basal se mantiene a los 3 meses en el $65 \%$ de los casos (14).

La situación basal se ha relacionado con la situación funcional al alta, de manera que está descrita peor evolución funcional al alta en los pacientes con un índice de Barthel previo menor de 60 (6). Así mismo, Abizanda et al. (6) ya sugieren un efecto suelo de este factor de riesgo, de manera que los que presentan una situación basal muy deteriorada o de dependencia total no pueden empeorar más. De forma opuesta, una buena situación previa es predictora de buena evolución funcional al alta. Así, en el trabajo de Zisberg et al. (11) aquellos pacientes sin DF basal fueron 2.5 veces más susceptibles de recuperar su situación previa en el momento del alta.

Existe poca bibliografía referida al deterioro funcional en Hospitalización a domicilio (HAD). Así, Shepperd et al. (15)no encontraron diferencias respecto a la hospitalización convencional en el seguimiento a 3 meses, mientras que Leff et al. (16) hallan mejores resultados funcionales en el grupo de HAD.

El presente estudio pretende analizar el deterioro funcional en los pacientes ancianos ingresados en una unidad de HAD y valorar los factores predisponentes.

\section{MÉTODOS}

Estudio observacional de los pacientes de 80 años o más ingresados en la Unidad de HAD de un Hospital Comarcal entre el 01/01/2018 y el 31/05/2019. 
Se excluyeron, al igual que en los estudios de Formiga et al. (14) y Mañas et al. (2), aquellos que fueron dados de alta antes de las 72 horas, los que ingresaban para tratamiento paliativo y los que fallecieron durante el ingreso, y aquellos con una situación funcional previa muy deteriorada (IB previo < 10). Tampoco se analizaron finalmente los pacientes que tuvieron que reingresar en el hospital como motivo de alta de HAD.

Se obtienen variables demográficas (sexo, edad) y sociofamiliares (lugar de residencia, cuidador principal), procedencia del ingreso (urgencias, hospitalización convencional, ingreso directo des de domicilio a petición de Atención Primaria o residencia de ancianos), duración del ingreso previo y del ingreso en HAD, variables clínicas (mantenimiento de sondaje urinario por más de 48 horas), comorbilidad (medida mediante el índice de comorbilidad de Charlson (17)).

Se solicitó consentimiento informado por escrito a todos los pacientes (familiares y/o cuidadores en el caso de deterioro cognitivo avanzado). Se garantizó el anonimato, la privacidad y la confidencialidad de los datos obtenidos. Se presentó y fue aprobado por la Comisión de Investigación del Centro.

Mediante entrevista con el paciente o el cuidador se recogió la situación funcional al ingreso en HAD, la situación basal (2 semanas antes del ingreso hospitalario) y al alta según la versión en catalán (18) del índice de Barthel (IB), (validada y equivalente a la versión en castellano), que se ha generalizado para valorar la situación funcional de los pacientes de edad avanzada, desde su publicación en 1965 (19). El IB consta de 10 ítems referidos a la autonomía del paciente para comer, lavarse-bañarse, vestirse, arreglarse, continencia de esfínteres, transferencias silla-cama, deambulación y uso de escaleras. Cada uno de estos ítems tiene una puntuación entre 0 y 15. El IB estratifica la dependencia funcional en: independencia para ABVD con una puntuación de 100, dependencia leve entre 60 y 95, dependencia moderada entre 40 y 55, dependencia grave entre 20 y 35 y dependencia total por debajo de 20.

Los datos de seguimiento se obtuvieron por teléfono 3 meses después del alta.

El análisis de datos se realizó con el paquete estadístico SPSS versión 15.0. Para la descripción de variables cuantitativas continuas se utilizó la media ( \pm desviación estándar), y las variables cualitativas se describieron mediante frecuencias expresadas en porcentaje. La comparación entre variables cuantitativas continuas se realizó mediante T de Student o pruebas no paramétricas cuando fue necesario. El análisis de frecuencias entre variables cualitativas se realizó mediante la prueba de chi-cuadrado o el test exacto de Fisher.

\section{RESULTADOS}

Ingresaron en el periodo de estudio 257 pacientes de edad igual o superior a 80 años, los motivos de exclusión se detallan en el diagrama de la Figura 1.

La mayoría (52.4\%) de los pacientes válidos para el análisis fueron hombres. Siendo significativamente mayores las mujeres, edad media 87.9 años $( \pm 5.2)$ respecto a los 85.7 años $( \pm 4.3, p=$ 0.0027 ) en los hombres. 45 pacientes (26.8\%) tenían $\geq 90$ años.

El principal origen de los ingresos fue el servicio de urgencias (71.4\%), el 19\% ingresó directamente desde su domicilio habitual sin atención previa en urgencias. Vivían en el domicilio familiar el $65.5 \%$ de los pacientes, 6 de ellos (5.5\%) disponían de cuidador privado las 24 horas del día, en el resto el cuidado era a cargo de los familiares. 
Figura 1: Motivos de exclusión

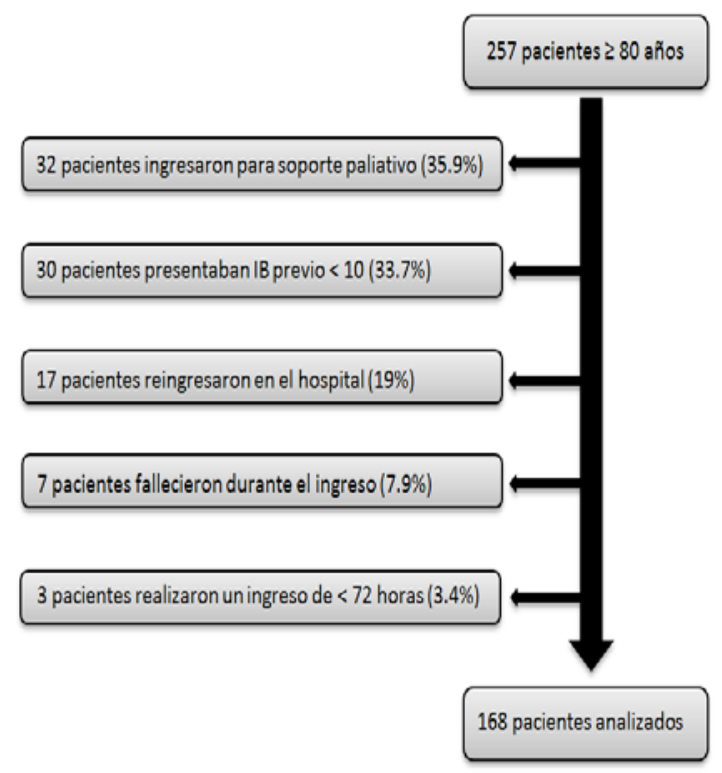

La situación funcional previa, al ingreso, al alta y a los 3 meses se recoge en la Tabla 1.

Tabla 1: Situación funcional previa, al ingreso, al alta y a los 3 meses

\begin{tabular}{|l|l|l|l|l|}
\hline & TOTAL & RG & DOMICILIO & \\
\hline IB previo & $64.17 \pm 28.49$ & $45.60 \pm 28.44$ & $73.95 \pm 23.25$ & $p<0.0001$ \\
\hline IB ingreso & $56.85 \pm 30.81$ & $37.59 \pm 27.98$ & $67.00 \pm 27.27$ & $p<0.0001$ \\
\hline IB previo - IB ingreso & $7.32 \pm 13.07$ & $8.02 \pm 11.62$ & $6.95 \pm 13.82$ & $p=0.62$ \\
\hline IB alta & $58.13 \pm 30.34$ & $38.88 \pm 27.78$ & $68.27 \pm 26.57$ & $p<0.0001$ \\
\hline IB previo - IB alta & $6.04 \pm 12.06$ & $6.72 \pm 11.94$ & $5.68 \pm 12.16$ & $p=0.59$ \\
\hline IB 3 meses & $61.00 \pm 31.06$ & $39.27 \pm 29.96$ & $72.34 \pm 25.11$ & $p<0.0001$ \\
\hline IB previo - IB 3m & $5 \pm 13$ & $7.71 \pm 14.36$ & $3.59 \pm 12.07$ & $p=0.07$ \\
\hline
\end{tabular}

RG: Residencia Geriátrica ; IB: Índice de Barthel 
Los pacientes procedentes de residencia geriátrica presentan peor situación funcional previa, al ingreso, al alta y a los 3 meses $(p<0.0001)$, sin diferencias significativas en la pérdida funcional al ingreso, al alta y a los 3 meses.

Presentaron pérdida funcional al ingreso (PFI) 68 pacientes (40.5\%), con una pérdida media de 18.1 \pm 15.11 puntos de IB.

Al alta, mejoraron el IB 2 pacientes (1.2\%), mantuvieron el previo $100(59.3 \%)$ y empeoraron respecto a la situación basal 66 sujetos (39.4\%).

A los 3 meses fallecieron 26 pacientes (15.5\%) y no se pudo contactar con $2(1.2 \%)$. De los 140 restantes, 18 (12.9\%) mejoraron su funcionalidad respecto a la previa, $67(47.8 \%)$ la mantuvieron, y en 55 (39.3\%) persistía la pérdida funcional tras el alta.

La evolución de la pérdida funcional al ingreso, al alta y a los 3 meses se recoge en la Tabla 2.

Como se refleja en la Tabla 3, las mujeres presentan peor situación funcional previa, al ingreso, al alta y a los 3 meses, sin que la diferencia en la pérdida funcional según el sexo sea significativa.

Un $44.7 \%$ de los pacientes de edad igual o superior a 85 años presentan deterioro funcional al alta respecto al $30 \%$ en los menores de 85 años $(p=0.042)$. Además, la pérdida funcional también es mayor en los mayores de 85 años, 8.13 puntos $( \pm 13.75)$ respecto 3.73 puntos $( \pm 8.13)$ en los menores $(p=0.05)$.

Tabla 2: Evolución funcional al ingreso, al alta y a los 3 meses

\begin{tabular}{|l|l|l|l|}
\hline Situación basal & Ingreso & Alta & 3 meses \\
\hline $\begin{array}{l}\text { Autónomos para ABVD } \\
\text { II 100) } \\
19(11.3 \%)\end{array}$ & $\begin{array}{l}\text { Mantienen IB } \\
16(84.2 \%)\end{array}$ & $\begin{array}{l}\text { Mantiene IB } \\
16(100 \%)\end{array}$ & Mantienen IB 9 (56.25\%) \\
\cline { 2 - 4 } & \multirow{2}{*}{$\begin{array}{l}\text { Empeora IB } \\
\text { (15.8\%) }\end{array}$} & $\begin{array}{l}\text { Mejora IB (recupera basal) } \\
1(33.3 \%)\end{array}$ & Mantiene IB 1 (100\%) \\
\cline { 2 - 4 } & $\begin{array}{l}\text { Eantiene IB } \\
1(33.3 \%)\end{array}$ & $\begin{array}{l}\text { Mejora IB (recupera basal) } 1 \\
(100 \%)\end{array}$ \\
\cline { 3 - 4 } & & $\begin{array}{l}\text { Empeora IB } \\
1(33.3 \%)\end{array}$ & Empeora IB 1 (100\%) \\
\hline
\end{tabular}




\begin{tabular}{|c|c|c|c|}
\hline \multirow[t]{18}{*}{$\begin{array}{l}\text { Dependientes para } \\
\text { ABVD } \\
\text { (IB 10-95) } \\
149(88.7 \%)\end{array}$} & \multirow[t]{7}{*}{$\begin{array}{l}\text { Mantienen IB } \\
84(56.4 \%)\end{array}$} & $\begin{array}{l}\text { Mejora IB (mejor que } \\
\text { basal) } \\
2(2.4 \%)\end{array}$ & $\begin{array}{l}\text { Mejora IB } \\
\text { (superior a basal) 1 (50\%) } \\
\begin{array}{l}\text { Empeora IB respecto al alta } \\
\text { (mantiene basal) } \\
1(50 \%)\end{array}\end{array}$ \\
\hline & & \multirow[t]{4}{*}{$\begin{array}{l}\text { Mantienen IB } \\
76(90.5 \%)\end{array}$} & Mejora IB 13 (17.1\%) \\
\hline & & & Mantiene IB 34 (44.7\%) \\
\hline & & & Empeora IB 20 (26.3\%) \\
\hline & & & $\begin{array}{l}9 \text { pérdidas a los } 3 \text { meses } 7 \dagger \\
(9.21 \%)\end{array}$ \\
\hline & & \multirow[t]{2}{*}{ Empeora IB 6 (7.1\%) } & $\begin{array}{l}\text { Mejora IB } 2(33.3 \%) \\
1 \text { mejor a basal }(50 \%)\end{array}$ \\
\hline & & & 4 † a los 3 meses (66.6\%) \\
\hline & \multirow[t]{11}{*}{$\begin{array}{l}\text { Empeora IB } \\
65(43.6 \%)\end{array}$} & \multirow[t]{4}{*}{$\begin{array}{l}\text { Mejora IB al alta } 28 \\
(43.1 \%) \\
\text { Recuperan basal } 7 \text { (25\%) }\end{array}$} & $\begin{array}{l}\text { Mejora IB } 10(35.7 \%) \\
1 \text { mejor a basal }(10 \%) \\
4 \text { igual a basal }(40 \%) \\
5 \text { inferior a basal }(50 \%)\end{array}$ \\
\hline & & & Mantienen IB 7 (25\%) \\
\hline & & & Empeora IB 7 (25\%) \\
\hline & & & 4 † 3 meses (14.3\%) \\
\hline & & \multirow[t]{4}{*}{ Mantienen IB 30 (46.1\%) } & $\begin{array}{l}\text { Mejora IB } 15(50 \%) \\
3 \text { mejor a basal }(20 \%) \\
11 \text { igual a basal }(73.3 \%) \\
1 \text { inferior a basal }(6.7 \%)\end{array}$ \\
\hline & & & Mantienen IB 5 (16.7\%) \\
\hline & & & Empeora IB 2 (6.6\%) \\
\hline & & & $8+3$ meses $(26.7 \%)$ \\
\hline & & \multirow[t]{3}{*}{$\begin{array}{l}\text { Empeora IB al alta } \\
\text { respecto al ingreso } 7 \\
(10.8 \%)\end{array}$} & $\begin{array}{l}\text { Mejora IB (no recuperan basal) } \\
3(42.8 \%)\end{array}$ \\
\hline & & & Empeora IB 1 (14.4\%) \\
\hline & & & $3+3$ meses $(42.8 \%)$ \\
\hline
\end{tabular}

ABVD: Actividades Básicas de la Vida Diaria ; IB: Índice de Barthel 
Tabla 3: Pérdida funcional según sexo

\begin{tabular}{|l|l|l|l|}
\hline & Mujeres & Hombres & \\
\hline IB previo & $55.69( \pm 29.13)$ & $71.88( \pm 25.71)$ & $p=0.0002$ \\
\hline IB ingreso & $43.38( \pm 28.94)$ & $66.36( \pm 29.49)$ & $p<0.0001$ \\
\hline IB ingreso - IB previo & $9.31( \pm 15.38)$ & $5.51( \pm 10.31)$ & $p=0.0596$ \\
\hline IB alta & $49.19( \pm 29.92)$ & $66.25( \pm 28.53)$ & $p=0.0002$ \\
\hline IB alta - IB previo & $6.50( \pm 13.08)$ & $5.63( \pm 11.11)$ & $p>0.5$ \\
\hline IB 3 meses & $52.61( \pm 29.74)$ & $68.70( \pm 30.43)$ & $p=0.0019$ \\
\hline IB 3 meses - IB previo & $4.70( \pm 14.25)$ & $5.27( \pm 11.84)$ & $p>0.5$ \\
\hline
\end{tabular}

IB: Índice de Barthel

La pérdida funcional al ingreso y al alta se relaciona con la mortalidad a los 3 meses. Los pacientes que presentan deterioro funcional al ingreso presentan una mortalidad a los 3 meses del $22.1 \%$ respecto al $11 \%$ de los que mantienen su situación basal al ingreso ( $p=0.043$ ), aumentando al $27.3 \%$ de los que presentan deterioro funcional al alta respecto al $7.8 \%$ en los que no presentan deterioro funcional al alta ( $p=0.0007$ ).

La pérdida funcional al alta es menor en los pacientes que no presentan ingreso hospitalario previo y en los que presentan una estancia hospitalaria previa al traslado a HAD de máximo 2 días, sin que las diferencias sean estadísticamente significativas (Tabla 4).

La estancia media fue de $9.49 \pm 4.18$ días. Los pacientes con estancia media $>7$ días presentan mayor pérdida funcional al alta que los pacientes con estancia media $\leq 7$ días (Tabla 4)

El uso de sonda vesical permanente o sondaje superior a 48 horas durante la estancia hospitalaria se asocia a peor resultado funcional al alta (Tabla 4). Sin embargo, no existen diferencias en el porcentaje de pacientes que presenta pérdida funcional al alta entre los que reciben sondaje vesical $(30.8 \%)$ y los que no (33.6\%). Los pacientes con sondaje superior a 48 horas durante el ingreso mantienen peor resultado funcional a los 3 meses (Tabla 4).

El índice de Charlson (IC) fue de $2.33 \pm 1.45$ (0-7), sin encontrar diferencias en la pérdida funcional al alta o a los 3 meses según comorbilidad en los pacientes estudiados. Tampoco se ha hallado relación con el IC respecto a la mortalidad intrahospitalaria ni a los 3 meses en los pacientes estudiados. 
Tabla 4: Pérdida funcional al alta según estancia hospitalaria previa, estancia media y uso de sonda vesical por más de 48horas durante el ingreso

\begin{tabular}{|c|c|c|c|c|}
\hline & $\begin{array}{l}\text { Número } \\
\text { pacientes }\end{array}$ & PFA & PF3m & \\
\hline Ingreso directo & 32 & $3.13( \pm 5.04)$ & & \multirow[t]{2}{*}{$p=0.13$} \\
\hline Ingreso previo & 136 & $6.73( \pm 13.10)$ & & \\
\hline $\begin{array}{l}\text { Ingreso previo } \\
\leq 2 \text { días }\end{array}$ & 125 & $5.40( \pm 11.17)$ & & \multirow[t]{2}{*}{$p=0.24$} \\
\hline $\begin{array}{l}\text { Ingreso previo } \\
>2 \text { días }\end{array}$ & 43 & $7.91( \pm 14.32)$ & & \\
\hline $\begin{array}{l}\text { Estancia media } \\
>7 \text { días }\end{array}$ & 105 & $7.71 \pm 14.40$ & & \multirow[t]{2}{*}{$p=0.0199$} \\
\hline $\begin{array}{l}\text { Estancia media } \\
\leq 7 \text { días }\end{array}$ & 63 & $3.25 \pm 5.62$ & & \\
\hline $\begin{array}{l}\text { Sonda vesical } \\
\text { permanente } \\
\text { o > 48horas }\end{array}$ & 26 & $12.31 \pm 14.58$ & & \multirow[t]{2}{*}{$p=0.0037$} \\
\hline $\begin{array}{l}\text { Sin sonda } \\
\text { por }>48 \text { horas }\end{array}$ & 142 & $4.89 \pm 11.23$ & & \\
\hline $\begin{array}{l}\text { Sonda vesical } \\
\text { permanente } \\
\text { o > 48horas }\end{array}$ & 21 & & $12.14 \pm 13.93$ & \multirow[t]{2}{*}{$p=0.0059$} \\
\hline $\begin{array}{l}\text { Sin sonda } \\
\text { por }>48 \text { horas }\end{array}$ & 119 & & $3.74 \pm 12.47$ & \\
\hline
\end{tabular}

PFA = Pérdida funcional al alta (IB previo - IB alta)

PF3m = Pérdida funcional a los 3 meses (IB previo - IB 3 meses)

PFA: Pérdida Funcional al Alta; IB: Índice de Barthel; PF3m: Pérdida Funcional a los 3 meses del alta

\section{DISCUSIÓN}

En el presente trabajo se analiza la DFH asociada al ingreso en HAD en pacientes mayores de 80 años. En el 39\% de los pacientes se objetiva deterioro de su situación basal y este porcentaje se mantiene a los 3 meses tras el alta. La mayor DFH al alta se asocia a una mayor mortalidad a los 3 
meses. La duración del ingreso hospitalario previo al ingreso en HAD superior a 2 días y ser portador de sonda urinaria más de 48 horas son factores asociados a un mayor DFH.

Existe gran variabilidad en la prevalencia de DFH entre los distintos trabajos publicados, debido principalmente a las diferencias en la población (edad, comorbilidad, situación funcional previa, etc.), en la metodología para medir el DFH y en las unidades de hospitalización estudiadas.

Entre las condiciones que se asocian a la aparición de DFH se encuentra la enfermedad aguda motivo de ingreso, que según varios estudios tiene escasa influencia por si sola en la aparición de DFH $(7,20)$, y solo el cáncer en el trabajo de Sager et al (10) se ha asociado con mayor riesgo de DFH. Son importantes también, en diferente grado según el paciente, la edad, el sexo, la comorbilidad, la polifarmacia, la situación socio-familiar, las alteraciones cognitivas, la situación funcional previa al ingreso, los tratamientos y pruebas diagnósticas, la utilización de sonda vesical durante más de 48 horas (12), el reposo en cama durante la hospitalización, la desnutrición o la aparición de infecciones nosocomiales (1,3-5).

Al igual que en otros estudios, la edad media de los pacientes incluidos en el presente estudio fue elevada $(2,6,8,9,12-14,21)$. A pesar de la edad avanzada, el porcentaje de pacientes que tenían una situación basal de independencia o dependencia leve para las ABVD coincidió con lo encontrado en otras poblaciones $(3,5,14,22,23)$.

La mayoría de los pacientes incluidos fueron hombres, a diferencia de lo hallado en la bibliografía $(3,5-9,13,14)$. Aunque la esperanza de vida es mayor en las mujeres, lo que justifica que supongan una proporción mayor de ingresos en hospitalización convencional, también son las que más viven solas, lo que supone que no dispongan de cuidador 24 horas, condición requerida para el ingreso en HAD.

Los mayores de 85 años son los que más capacidad funcional pierden, en concordancia con lo hallado en la bibliografía consultada $(3,10,21,23)$.

La incidencia de PFI es menor en nuestro estudio (40.5\%) que en la bibliografía consultada $(7,9,11,12)$ probablemente como sesgo de selección, al no aceptar el traslado a HAD los familiares de pacientes con mayor PFI que residen en domicilio por verse sobrecargados. De hecho, la PFI es mayor (aunque no estadísticamente significativa) en los pacientes procedentes de residencia, en los que no cabe esperar dicho sesgo, ya que disponen de cuidador profesional.

A diferencia de la bibliografía consultada, en la que los hombres obtienen mejores resultados funcionales que las mujeres tras la hospitalización $(8,10,11,13,23,24)$, no hemos hallado diferencias en la situación funcional al alta, probablemente por el tamaño muestral.

Los pacientes que sufren DF prehospitalización (DF al ingreso respecto a la situación basal) presentan peor evolución funcional durante la hospitalización, con menores tasas de recuperación funcional al alta, igual que lo encontrado en la bibliografía (1,3,6,14,20), persistiendo la relación con el DF en nuestra serie hasta los 3 meses, hasta el mes según Alarcon et al. (1) y Gutiérrez et al. (20). En nuestra serie, el DF se relaciona con la mortalidad a los 3 meses, habiéndose descrito relación con el DF con la mortalidad al alta y al mes según Abizanda et al. (6), a los 6 meses según Alarcon et al. (1) y al año según Boyd et al. (8)).

El porcentaje de defunciones durante el ingreso en HAD fue menor al registrado en estudios previos $(1,6)$, incluso si se valoran las muertes durante el presente ingreso en los pacientes no analizados por IB previo inferior a 10 o reingresos hospitalarios, probablemente por no ingresar habitualmente en HAD los pacientes de mayor riesgo vital (enfermedad cerebral aguda, cardiopatía isquémica, sepsis grave (8)) a no ser que se consensue al traslado con la familia un manejo paliativo.

El factor de riesgo más importante susceptible de ser modificado es la inmovilidad, se conoce que la inmovilidad durante el ingreso tiene un impacto negativo sobre la funcionalidad, apareciendo de forma muy temprana y siendo evidente tras 48 horas de reposo en cama (21), por lo que el DFH se presenta incluso en estancias cortas $(14,22)$. Los pacientes con estancias previas cortas y estan- 
cia media inferior a una semana presentan mejor resultado funcional, sin que se haya encontrado diferencia en los pacientes que ingresan directamente desde el domicilio por una representación muy pequeña en la muestra.

A diferencia de Vidan et al. (12), que encontraron que el $77.1 \%$ de los pacientes que usaron sonda vesical durante $>48$ horas presentó DFH, frente al $50.4 \%$ de los que no mantuvieron sondaje durante la hospitalización, no hallamos diferencias en el porcentaje de pacientes con DFH, aunque si con la magnitud de la pérdida funcional, que se mantiene a los 3 meses.

No hemos comparado el DFH con los pacientes ingresados en la planta de hospitalización convencional, pero los resultados respecto a la bibliografía consultada apuntan a una menor DFH en HAD respecto al ingreso convencional.

En cuanto a las limitaciones del presente estudio destacamos el tamaño muestral y la ausencia de un grupo control hospitalario. Son necesarios estudios con mayor población y la presencia de un grupo de comparación en otras situaciones de hospitalización así como en diferentes zonas geográficas y organización de HAD para poder generalizar los resultados. No obstante, los resultados apuntan a un beneficio de HAD en cuanto a la minimización de la DFH en pacientes mayores de 80 años.

Como conclusiones destacan los pacientes institucionalizados presentan peor situación funcional que los pacientes procedentes de domicilio. La edad > 85 años y el sexo femenino se asocian a peor resultado funcional al alta. La pérdida funcional se relaciona con la mortalidad a 3 meses. Los pacientes con ingreso convencional $\geq 2$ días y estancia media superior a 7 días sufren mayor pérdida funcional. El sondaje vesical permanente $0>48$ horas supone peor resultado funcional al alta y se mantiene a los 3 meses.

\section{BIBLIOGRAFÍA}

1. Alarcón T, Bárcena A, González-Montalvo JI, Peñalosa C, Salgado A. Factors predictive of outcome on admission to an acute geriatric ward. Age Ageing. 1999;28(5):429-32. DOI: 10.1093/ageing/28.5.429

2. Mañas MD, Marchán E, Conde C, Sánchez S, Sánchez-Maroto T, Molina MC. Deterioro de la capacidad funcional en pacientes ancianos ingresados en un Servicio de Medicina Interna. An Med Interna. 2005;22(3):130-2. DOI: 10.4321/s0212-71992005000300006

3. Covinsky KE, Palmer RM, Fortinsky RH, Counsell SR, Stewart AL, Kresevic D, et al. Loss of independence in activities of daily living in older adults hospitalized with medical illnesses: Increased vulnerability with age. J Am Geriatr Soc. 2003;51(4):451-8. DOI: 10.1046/j.15325415.2003.51152.x

4. Osuna-Pozo CM, Ortiz-Alonso J, Vidán M, Ferreira G, Serra-Rexach JA. Revisión sobre el deterioro funcional en el anciano asociado al ingreso por enfermedad aguda. Rev Esp Geriatr Gerontol. 2014;49(2):77-89. DOI: 10.1016/j.regg.2013.08.001

5. Covinsky KE, Justice AC, Rosenthal GE, Palmer RM, Landefeld CS. Measuring prognosis and case mix in hospitalized elders: The importance of functional status. J Gen Intern Med. 1997;12(4):203-8. DOI: 10.1046/j.1525-1497.1997.012004203.x

6. Abizanda-Soler P, León-Ortiz M, Romero-Rizos L, Sánchez-Jurado PM, Luengo-Márquez C, Domínguez-Martín L, et al. La pérdida funcional al ingreso, principal variable explicativa de discapacidad y mortalidad al alta y al mes en ancianos hospitalizados. Rev Esp Geriatr Gerontol. 2007:42(4):201-11. DOI: 10.1016/S0211-139X(07)73552-7 
7. Landefeld CS, R M Palmer, D M Kresevic, R H Fortinsky, J Kowal. A randomized trial of care in a hospital Medical unit especially designed to improve the functional outcomes of acutely ill older patients. NEngJMedicine. 1995 May 18;332(20):1338-44. DOI: 10.1056/NEJM199505183322006

8. Boyd CM, Landefeld CS, Counsell SR, Palmer RM, Fortinsky RH, Kresevic D, et al. Recovery in Activities of Daily Living Among Older Adults Following Hospitalization for Acute Medical IIIness. J Am Geriatr Soc. 2008;56(12):137-52. DOI: 10.1111/j.1532-5415.2008.02023.x

9. Ramos-Cortés MR, Romero-Pisonero E, Mora-Fernández J, Silveira-Guijarro LJ, Ribera-Casado JM. Análisis de mortalidad tras el ingreso en una unidad de agudos de geriatría: Influencia de la dependencia funcional. Rev Esp Geriatr Gerontol. 2007;42(4):212-7. DOI: 10.1016/S0211139X(07)73553-9

10. Sager M, Rudberg M, Jalaluddin M et al. Hospital admission risk profile (HARP): identifying older patients at risk for functional decline following acute medical illness and hospitalization. J Am Geriatr Soc. 1996;44:251-7. DOI: 10.1111/j.1532-5415.1996.tb00910.x

11. Zisberg A, SHADmi E, Sinoff G, Gur-Yaish N, Srulovici E, Admi H. Low mobility during hospitalization and functional decline in older adults. J Am Geriatr Soc. 2011;59(2):266-73. DOI: 10.1111/j.1532-5415.2010.03276.x

12. Vidán-Astiz MT, García ES, Armesto MA, Errasquin BM, De La Casa AM, Ortiz FJ, et al. Deterioro funcional durante la hospitalización en ancianos. Beneficios del ingreso en el servicio de geriatría. Rev Esp Geriatr Gerontol. 2008;43(3):133-8. DOI: 10.1016/s0211-139x(08)71172-7

13. Condorhuamán-Alvarado PY, Menéndez-Colino R, Mauleón-Ladrero C, Díez-Sebastián J, Alarcón T, González-Montalvo JI. Factores predictores de pérdida funcional al alta en ancianos hospitalizados por enfermedad aguda. Rev Esp Geriatr Gerontol. 2017;52(5):253-6. DOI: 10.1016/j. regg.2017.03.006

14. Formiga F, Jacob X, Vidal M, Soto AL, Masanés F. Evaluation of functional capacity after hospital admission of nonagenarian patients. Med Clin (Barc). 2000;115(18):695-6. DOI:10.1016/S00257753(00)71666-6

15. Shepperd S, Harwood D, Jenkinson C, Gray A, Vessey M, Morgan P, et al. Randomised controlled trial comparing hospital at home care with inpatient hospital care. I: three month follow up of health outcomes. BMJ. 1998 Jun 13;316(7147):1786-91. DOI: 10.1136/bmj.316.7147.1786.

16. Leff B, Burton L, Mader SL, Naughton B, Burl J, Greenough WB, et al. Comparison of functional outcomes associated with hospital at home care and traditional acute hospital care. J Am Geriatr Soc. 2009;57(2):273-8. DOI: 10.1111/j.1532-5415.2008.02103.x

17. Charlson ME, Pompei P, Ales KL, MacKenzie CR. A new method of classifying prognostic comorbidity in longitudinal studies: development and validation. J Chronic Dis. 1987;40(5):373-83. DOI: 10.1016/0021-9681(87)90171-8

18. Aranzana-Martinez A, Borras-Gallat E, Carrés-Esteve L, Cegri-Lombardo F, Figueriras-Novelle L, Garolera-Bruguera D, et al. Salut a casa: Valoració integral [monografía en Internet]. Barcelona: Institut Català de la Salut: 2004 [consultado 18 agosto 2020]. Disponible en: https://docplayer. es/16601317-Valoracio-integral-institut-catala-de-la-salut-institut-catala-de-la-salut.html

19. Mahoney F, Barthel D. Functional evaluation: The Barthel Index. Md State Med J. 1965;14:61-5. PMID: 14258950

20. Gutiérrez-Rodríguez J, Domínguez-Rojas V, Solano Jaurrieta. Functional deterioration secondary to hospitalization for an acute disease in the elderly. An analysis of its incidence and the associated risk factors. Rev Clin Esp. 1999 Jul;199(7):418-23. PMID: 10481556 
21. Hirsch C, Sommers L, Olsen A, Mullen L, Hunter-Winograd C. The Natural History of Functional Morbidity in Hospitalized Older Patients. J Am Geriatr Soc. 1990;38(12):1296-303. DOI: 10.1111/j.1532-5415.1990.tb03451.x

22. Córcoles-Jiménez MP, Ruiz-García MV, Saiz-Vinuesa MD, Muñoz-Mansilla E, Herreros-Sáez L, et al. Deterioro funcional asociado a la hospitalización en pacientes mayores de 65 años. Enferm Clin. 2016;26(2):121-8. DOI: 10.1016/j.enfcli.2015.09.010

23. Novo-García C, Lafuente-López C, Romero-Arancón C. Deterioro funcional del anciano tras un ingreso hospitalario. Rev Enferm CyL. 2009;1(2):20-5.

24. Cruz-Lendínez A.J, Pancorbo-Hidalgo, PL, García-Fernández FP, Carrascosa-García MI, Jiménez-Díaz MC, Villar-Dávila R. Deterioro funcional en ancianos ingresados en un hospital sin unidades geriátricas. Gerokomos. 2010;21(1):8-16. 